\title{
New And/Or Interesting At The MSA '95 Conference
}

As is our custom, and hopefully in the interest of readers who were not able to attend the MSA '95 Conference, we have attempted as follows to summarize what was "New And/Or Interesting.

- $4 \mathrm{pi}$ Analysis offered several scientific Macintosh ${ }^{\circ}$ and Power Macintosh ${ }^{\text {TM }}$-based product packages and tools for the microscopist. 4 pi's systems provide digital image acquisition, $x$-ray spectrum acquisition, spectral image mapping, and direct writing capabilities for desktop microscopy systems. The 4pi acquisition hardware is based on state-of-the-art DSP technology providing 120 MIPS processing in a pipelined architecture. Systems include software plug-ins for a variety of commercial image and $x$-ray analysis programs. Custom processing software is available. 4pi analysis: (919)489-1757. Fax: (919)4891487

- Alden Electronics has announced the availability of a Windows driver for its Continuous Tone Printer, the 9315CTP. The new software driver allows any Windows-based program, such as MS Powerpoint or Adobe Photoshop to output to the printer. The 9315CTP provides 256 gray scale printing on a 10.1 inch-wide image for photographic quality hard copy. This quality makes the $9315 \mathrm{CTP}$ ideal for applications where a high quality image is needed. The printer uses economical thermal technology which keeps per-print costs as low as nine cents per piece. With just three moving parts, the printer has a Mean Time Between Failures of 20,000 hours, equivalent to about 10 years of full-time printing. It takes output from PC's, Macs, Sun and other workstations, and various imaging systems. Alden Electronics: (508)366-8851, Fax: (508)898-2427.

- AMRAY, INC. announced its new line of 3000 Series Scanning Electron Microscopes. The new product line includes the Model 3200 ECO Variable Pressure SEM and the Low Energy Advanced Performance Model 3600 LEAP SEM. The Model 3600 LEAP, on display at MSA, boasts of a high resolution specification of $4 \mathrm{~nm}$ at $1 \mathrm{KV}$, and $1.5 \mathrm{~nm}$ at $15 \mathrm{KV}$. Other features include a $2048 \times 2048$ frame buffer, embedded computer control of all SEM functions, and patented Schottky Field Emission for uncompromised analytical performance (EDSNDS). Amray, Inc: (617)275-1400, Fax: (617)275-0740.

\section{- AutoQuant Imaging Inc. announced the following new products:}

1. AutoDeblur ${ }^{\mathrm{TM}}$ - a statistically-optimal and full-featured 3-D Blind Deconvolution software package for confocal, wide-field fluorescence, and transmitted-light brightfield microscopies $(\$ 4,995)$.

2. Auto3DCollect ${ }^{T}$ - a low-cost PC based 3-D imaging system for widefield fluorescence and transmitted-light brightfield microscopies $(\$ 2,995)$.

3. VibraSorb ${ }^{\mathrm{TM}}$ - a low-cost modular vibration attenuation system (\$199/stage).

AutoQuant Imaging, Inc:: (518)276-2138, Fax: (518)276-6380

- CamScan USA Inc. introduced many new products to augment their line of SEMs. The new EnVac low vacuum SEM allows imaging and analysis of non-conductive samples such as plastics, ceramics, and biologicals without any sample preparation. Users can switch between normal high vacuum and EnVac modes in seconds with a single pushbutton. Also introduced is the CamScan OrTex, a complete system from one manufacturer for obtaining macro-structural information and automated orientation imaging in the SEM. OrTex utilizes CamScan's FASTRAC computer-controlled absolute positioning stage, and beam control, to provide very high speed EBSP/texture analysis automation. Data acquisition rates of more than 60 measurements/second can be easily achieved. CamScan USA: (412)772-7433, Fax: (412)772-7434

- The Carl Zeiss, Inc. exhibit featured the DSM 982 GEMINI Field-Emission SEM \& the EM 910 TEM, both with unique features. The DSM 982 GEMINI offers a patented magnetic/electrostatic objective lens that provides exceptional imaging \& resolution capabilities, especially at low beam energies $(e . g .1 \mathrm{~nm}$ resolution at $30 \mathrm{keV}$ ). The EM 910 , for the first time, incorporates the Koehler illumination principle in an electron microscope, resulting in optimum image contrast \& snarp reduction in specimen irradiation damage. It also allows for direct switching from TEM to STEM operation. Zeiss confocal microscopy specialists also demonstrated the latest software for confocal imaging with the Zeiss LSM

\section{Carl Zeiss, Inc: (800)356-1090, Fax: (914)681-7443.}

- Denton Vacuum, Inc. introduced the newest in their Explorer TM series of table top units, the Desk II Turbo Sputter Coater. The Desk II TSC is a totally self-contained turbo molecular pumped sputtering unit. Its integral mechanical pump with on-board $801 / \mathrm{s}$ turbo pump assures faster and safer operation and takes up less table space than systems with awkward floor-mounted pumps. The Desk II TSC comes ready-to-operate with all the required pumps and a starter gold target. The Desk II TSC's unique design is the most advanced in the industry. Lighted push buttons arranged logically by function provide sure, easily reproducible films. To monitor gas pressure and electrical current, the Desk II TSC uses reliable solid state electronic bar graphs instead of analog meters. This allows more accurate readings and increases run-to-run repeatability of the deposited films. The Denton Desk II TSC sputters the typical gold, goldpalladium and platinum films in common use today as well as the newer high resolution materials such as chromium and tungsten for use with today's FESEM. Denton Vacuum, Inc.: (609)439-9100, Fax: (609)439-9111

- Diatome U.S. had on display their entire range of diamond knives including their Ultra-Thin, Semi-Thin (for alternating sectioning/HVEM), Cryo Wet/Dry, Histo (for sectioning 1-10 micron sections) and their Cryo Histo. Also on display was the Static Line, a unique static eliminator for wrinkle-free cryo ribbons, and the Diamond Trimming Tool. Introduced at the meeting was the availability of a $10 \mathrm{~mm}$ Histo Diamond Knife which in the past has been available in 4,5 , and 6 $\mathrm{mm}$. The unique Diatome Diamond Knife exchange program was announced whereby you trade in an old knife from a competitor and get a brand new Diatome, any size, and simply pay the resharpening price of the size that you choose. Diatome, U.S.: (215)646-1478/(800)523-5874, Fax: (215)646-8931

6 Digital Instruments displayed its versatile Dimension ${ }^{\text {Th }} 3000$ Scanning Probe Microscope and ran samples on the floor for nearly twenty customers. DI featured Phase Contrast Imaging, which, for many of the samples, revealed details of compositional transitions not found in the topographic AFM images. The phase contrast data is acquired at the same time as topographic data and is recommended for a wide variety of materials. An application note on this topic is available on request from Digital Instruments at (800)873-9750, Fax: (805) $899-3392$

- E.A. Fischione Instruments, Inc. has introduced the revolutionary Model 1400 Plasma Cleaner. The Model 1400 applies a low-energy, High Frequency (HF) plasma that effectively removes amorphous damage from TEM specimens and eliminates contamination from both the specimen and the TEM Specimen Holder. Also introduced was a series of Vacuum Storage Containers from TEM Specimen Holders to enable ultra-clean specimen/specimen holder storage. E A. Fischione Instruments, Inc.: (412)325-5444, Fax: (412)325-5443.

- The Eastman Kodak Company presented a complete line of products for the capture, output, management and storage of both digital and traditional silver halide images. New this year were the EOSDCS 5 camera for digital capture, XLS 8600 printer for photographic quality output, and PCD Writer 225 for desktop storage of up to $680 \mathrm{MB}$ of data onto a single CD-ROM. In addition, Kodak introduced their WWW site (http://wWw kodak com), providing an on-line source of product information, application descriptions and sample images. Eastman Kodak Company: (716)726-7919. Fax: (716)726-0818.

- Edax International introduced the newest product in its range of $x$-ray microanalysis systems running in Microsoft's Windows 95 . The $D X^{\text {Prime }}$, as it is known, also includes a $90 \mathrm{MHz}$ Pentium $\mathrm{CPU}$ and new analytical software. Also introduced was improved $\mathrm{x}$-ray detector performance to increase the peak/background and improve the sensitivity of the Edax range of detecting units for SEMs and TEMs. Paul Oravetz, Edax International: (201)529-6277, Fax (201) $529-3156$ 
- Electrolmage Corporation demonstrated the MicroLumina camera and the EICAS-D digital imaging workstation. The MicroLumina is an affordable, digital camera with $35 \mathrm{~mm}$ film resolution. The MicroLumina captures color images 36 bits deep at up to $3380 \times 2700$ pixels making it an excellent instrument for micro and macro photography and image duplication. The EICAS-D maximizes the potential of the MicroLumina camera by displaying images at $1600 \times 1280$ in 24-bits. EICAS-D also includes a substantial image analysis and processing package, imaging database, and removable archiving. Electrolmaging: (516)773-4305, Fax: (516)773-2955.

- Electron Microscopy Sciences introduced their 1995 Series XII catalog for the first time. Included in the catalog are 400 pages of the most up-to-date chemicals, supplies, accessories, and equipment for the fields of microscopy (materials as well as biological), histology, and general lab research. Also introduced for the first time was the EMS Portable Cryo Fixation Gun: the only unit currently available that can be used for both in situ and routine cryo techniques. Electron Microscopy Sciences: (215)646-1566, Fax: (215)646-8931.

- Ernest F. Fullam, Inc. in cooperation with Princeton Instruments, introduced an upgraded TEM CCD camera system. The new Princeton camera features a cooled CCD with a $1317 \times 1035$ pixel array and 12 bit resolution. Camera system software supports virtually all PC or Mac based computers. The camera interfaces are available for all modern TEMs and feature a choice of YAG or phosphor detectors. Ernest F. Fullam, Inc.: (518)785-5533, Fax: (518)785-8647.

- ETP-USA exhibited its Series IV Robinson Backscattered Electron Detector. The microprocessor controlled BSE detector, the original with Windows capability, was met with great acceptance. The ETP-USA SEM Chamber View System was also on display and enjoyed equal success. ETP-USA introduced its new 5 year warranty at the meeting. Enjoying over 10 years of success, ETP.USA's products could be seen all over the exhibition floor on many different models of SEMS. ETP-USA: (510)449-8534, Fax: (510)449-8996.

- Evex Analytical Instruments demonstrated WinEDS, a low cost and easy-to-use PC-Windows, high performance, $X$-ray microanalysis system. Also shown was VIDX Interfazer (to transfer Tracor \& PGT spectra \& images at high speed to a PC), VIDX Vision (lab networking system) and VIDX SCAN CCD (1536 x 10241.6 mega pixel array, 14 bit, cooled scientific grade CCD with square pixel and zero defects, with utra-low noise electronics, high performance low light levels and high dynamic range). Evex Analytical Instrusments: (908)874-3800, Fax: (908)874-4647.

- FEl Company featured its $\mathrm{LaB}_{4}$ and $\mathrm{CeBix}{ }^{\mathrm{TM}}\left(\mathrm{CeB}_{6}\right)$ Mini Vogel Mount (MVM) cathodes as well as its field emission cathodes and focusing columns. FEl's MVM was the first true long-life high stability $\mathrm{LaB}_{6}$ cathode. FEl's CeBix MVM was installed in both Topcon's SM510 (with wet SEM 3D attachment) and ElectroScan's Environmental ESEM 2020 for equipment demonstrations during MSA. Also presented was FEl's Focused Ion Beam (FIB) Workstation for SEM and TEM specimen preparation and scanning ion microscopy. FEI Company: (503)640-7500, Fax: (503)640-7509.

- Gatan demonstrated two key new products at the Kansas City MSA meetIng. 1) The new GIF software offers an intelligent ICON driven user interface with many new automated functions, and operation from a single computer. 2) Gatan's new MegaScan ${ }^{\mathrm{TM}}$ camera features a $2 \mathrm{~K} \times 2 \mathrm{~K}$ CCD with variable speed readout. Operating in the TurboScan mode it can readout $2.5 \mathrm{M}$ pixels/second, or using the View mode you get large photographic quality images. Gatan. (510)463-0200, Fax: (510)463-0204.

- IXRF Systems, Inc. introduced, complete with Si detectors, the first EDS/Imaging/X-ray mapping system at $1 / 2$ the cost of leading EDS manufacturer's systems. It's powerful quantitative EDS package combined with point analysis, digital imaging, and X-ray mapping, provides a user friendly system with most key features of the the larger EDS companies. IXRF Systems, Inc.: (713)286-6485, Fax: (713)286-2660.

- Kevex instruments has made improvements in the GUI at each level of ithe Sigma Analyzer, while adding a level 4 system. The major GUI changes nave been related to the imaging levels, as the GUI has been made more intu- itive and unique processing features have been added. The level 4 Sigma now has FFT, Image Stitching, Locked \& Liberated Analysis, and Nearest Neighbor Analysis to mention just a few of the new features. Digital Multipoint Analysis (DMA) has been optioned to all Sigma level analyzers, enabling macro capability with unique graphic outputs for qualitative and quantitative line profiles. These features, along with the latest Integration / Column Control (ISEM) make various modes of automation standard on the new level 4 , with the latest in Automated Image Analysis $\left(\mathrm{AlA}^{2}\right)$. Kevex Instruments: (805)295-0019, Fax: (805)295-8714.

- Leica, Inc. demonstrated the LEICA S400i SEM, fully integrated with the Link ISIS X-ray analysis system and the new LEICA S430 o SEM integrated analytical package combining the LEICA S430 SEM with the Kevex Sigma Microanalyzer. Leica also showed the new UItracut T/FCS, AFS (automatic freeze substitution), Knifemaker, Ultrastainer and Lynx EM tissue processor. The TCS $4 \mathrm{D}$ confocal microscope and VT 1000 vibrating blade microtome were also displayed. Leica, Inc: (800)248-0123/(708)317-7205, Fax: (708)405-0030.

- Materials Analytical Services offers custom instrumentation and accessories engineering for E-Beam and related instruments. Researchers, engineers, and/or technical personnel can call to discuss modifications or design of existing and new devices for TEMs, SEMs and other instruments. Our specialists are experienced in the modification of whole systems including lenses, power supplies, vacuum pumps, electronics, and mechanical components. The high speed BB6000J Electrostatic Beam Blanker and 120 s High Voltage SEM for lithographic and semiconductor groups are available engineered products. Materials Analytical Services: (800)421-8451, Fax: (404)368-8256.

- Oncor Imaging demostrated both Macintosh and PC based image analysis for a wide variety of primarily microscopy based scientific applications. These capabilities included technologies for both high resolution monochrome and HSI true color image analysis with accompanying extensive tool boxes for image processing and object measurement. Data and images from the analyses can be output in standard formats that allow for easy transfer to other software packages or computer systems. Using the available scripting and macro languages, the Oncor Imaging image analysis systems also have the flexibility to be configured to the user's specific applications or be re-adapted to future applications. Oncor Imaging: (301)990-0100, Fax: (301)990-8391.

- OXFORD Instruments displayed their $L N_{2}$ free EDX Detector on a LEICA 440. The detector uses a patented closed cycle cooling technique to achieve the temperataures necessary for the proper performance of an X-Ray detector. The detector had an on-column resolution of $129 \mathrm{eV}$ at MnKa and $68 \mathrm{eV}$ at FKa. Because the Link Ultracool Detector requires no regular maintenance a customer needs only to plug it in to an electrical outlet and forget it. In addition, Oxford demonstrated on the LEICA 440, with Microspec, the integrated EDS $N D S$ system, THETA, and the LINK OPAL Crystallography System. OXFORD Instruments: (508)369-9933, Fax: (508)369-8287.

- Philips Electronic Instruments displayed the CM120 BioTWIN, part of the Philips CM Series of TEMs. 'CM' stands for "customized microscopy," a concept which reflects Philips' orientation toward indiviidual customer requirements. Each CM120 BioTWIN is tailored to its owner. Philips supports the customer with hands-on training, service support, applications support, and software updating. Philips Electronic Instruments: (201)529-6168, Fax: (201)529-2252.

- Princeton Gamma-Tech introduced IMIX-PTS with Position Tagged Spectrometry, a new and revolutionary method for microanalysis data collection. While rapidly scanning the sample and acquiring a high resolution electron image, $X$-ray counts are processed and encoded with the specimen $X, Y$ coordinate information. In one efficient collection, the operator gets a high resolution digital image, a full EDS spectrum from the image area, multiple spectra from any regions selected within the image and quick $X$-ray maps for all elements in the periodic table. The maps and spectra may be displayed live, during collection, or may be requested after collection from stored position tagged data. Princeton Gamma-Tech: (609)924-7310, Fax: (609)924-1729.

Continued on next page 
- RJ Lee Instruments exhibited its latest generation of the PERSONAL SEM@ featuring the new Remote Diagnostics Package which allows a service engineer to diagnose problems on the PERSONAL SEM from a remote location; off-line data processing software; and the Stereoptical Preview and Relocation Station wherein the user can locate a series of features of interest with a light microscope and then easily relocate them in the PERSONAL SEM without remounting the specimen. RJ Lee Instruments: (412)744-0100, Fax: (412)7440506 .

- The CELLscan system, from Scanalytics, is the digital alternative to confocal microscopy which provides the ability to image delicate living specimens using conventional fluorescence optics with resolution in $X, Y$, and $Z$ which is superior to the signal rejection technique of confocal microscopy. The heart of the system is the patented EPR algorithm which was developed by Dr. Fredric S. Fay and the Biomedical Imaging Group at the University of Massachusetts Medical School (US Patent \# 5047968) which effectively reassigns all out-of-focus signal present in a through-focus volume to their proper places of origin. Scanalytics: (508)663-7598 or (800)325-3110, Fax: (508)663-0150.

- South Bay Technology, Inc. introduced several new sample preparation systems including the IV3 Ultra Low Angle lon Mill. The IV3 is produced in Hungary and was developed by Dr. Arpad Barna of the Hungerian Institute for Technical Physics. A short workshop was given by Dr. Barna which demonstrated the high power Teletwin ion guns and how effectively they work with the sample biasing facility for ion milling at near $0^{\circ}$. Also demonstrated at the workshop was

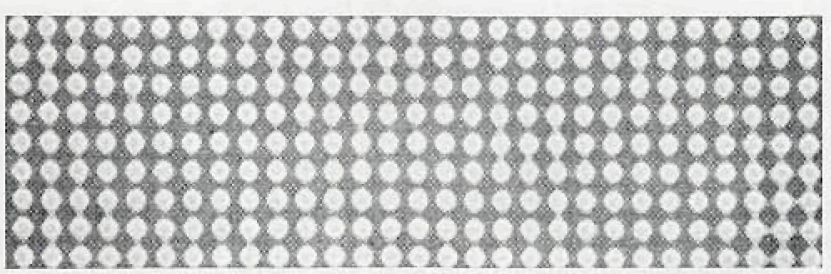

\section{Submicron Calibration Standards}

\section{$0.3 \mu \mathrm{m}$ 2-Dimensional Grid Order an MXS 302CE}

Each An 0riginal

Tungsten over Polymer on Silicon

Accurate

$3 \sigma$ variation $\pm 3 \%$ over $3 \mathrm{~mm} \times 4 \mathrm{~mm}$ area

Durable

over $1,000,000$ new measurement sites per sample

Easy to Use

Quick and Easy Imaging Characteristies

Versatile

Useful from $10,000 \mathrm{X}$ to over $100,000 \mathrm{X}$

Inexpensive

$\$ 415$ each

Available Today!

Ask for the New MOXTEK Calibration Standard from your favorite microseopy supplier or

\section{MOXTEX, Ine. (801)225-0930}

\section{Try 0ne NOW Risk Free}

Like it or send it back for a full refund
SBT's new Tripod Polishing System. South Bay Technology: (714)492-2600, Fax: (714)492-1499.

- SPI Supplies introduced its new and improved Stain "n Wash Grid Staining System (SPI \#02480-AB) for the efficient staining of TEM grids (up to 100 at a time in 30 minutes). In addition to the primarily glass (as opposed to plastic) contruction, the unit is designed to use extremely small volumes (2-6 ml) of staining reagents, a real "plus" when using expensive immuno reagents. It was designed for most heavy metal post-staining techniques of thin sections as well as immunochemical labeling or enzyme localization techniques. Contact us on the WWW at <http//mail.cccbi.chester.pa.us/spi/new/stanwash html>. SPI Supplies: (610)436-5400, Fax: (610)436-5755.

- Ted Pella, Inc. displayed its latest Microwave Specimen Preparation System and presented a well attended tutorial on 3-hour Tissue Preparation for TEM. Also on exhibit was the PhotoPro 2000 Gray Scale Printer which can produce dry continuus tone black/white prints digitally. PhotoPro accurately reproduces digitally processed, transmitted or stored data including active memory, optical disk, tape or hard disk. The image can be acquired via an SEM or TEM, CCD camera or scanner, and the digital picture is manipulated with IBM PC compatible or Macintosh computers. Ted Pella, Inc.: (916)243-2200, Fax: (916)243-3761.

- TexSEM Laboratories, Inc. had its Orientation Imaging Microscopy ${ }^{\text {TM }}$ $\left(O \mid M^{T M}\right)$ system in live operation on a Philips XL-30 SEM in the NORAN booth. Although several OIM systems are installed around the world, this was the first live demo of OIM at an industry conference. It was also the unveiling of the TSL OIM software on NORAN's SunNoyager platform, allowing materials scientists to perform both chemical and crystallographic characterization on a single platform. Dr. David Dingley, TSL's Chief Scientist, presented two seminars on OM which were well attended (over 100 people). In addition, three papers were presented at the conference by OlM users. Dr. Roger Alvis of Advanced Micro Devices discussed the use of OIM to analyze aluminum interconnects on integrated circuits; Dr. Hasso Weiland of Alcoa presented his OlM results for aluminum processing; and Dr. Hamid Garmestani of Florida State University analyzed aluminum-lithium superplastic alloys using OIM. TexSEM Laboratories, Inc: (801)344-8990, Fax: (801)344-8997.

- Topcon Technologies Inc. showed its new model 520 FE SEM that makes field-emission performance available at $\mathrm{LaB}_{\overline{0}}$ prices. The $520 \mathrm{FE}$ SEM offers extremely high spatial resolution and is ideal for a wide range of SEM applications - especially for those requiring high-resolution and low $\mathrm{kV}$. Topcon also exhibited its new versatile model 510 SEM that can be purchased as a stand-alone SEM or as an SEM/EDX system. Both systems come with Topcon's unique Dual Control ${ }^{\mathrm{TM}}$ feature that combines manual and computer operation for truly easy and convenient operation. Topcon Technologies, Inc. (201)261-5410, Fax: (201)262-1504.

- TopoMetrix demonstrated the Observer ${ }^{\mathrm{TM}}$ SPM for integration into SEMS ands the Aurora ${ }^{T M}$ near-field scanning optical microscope (NSOM). The Observer allows you to have both the higher magnification of an SPM and a variety of proximal probe analysis techniques plus a high-resolution SEM in one integrated system. The Aurora NSOM offers all of the advantages of optical microscopy plus the extremely high resolution $(<50 \mathrm{~nm})$ of an SPM. The Aurora NSOM is the first commercial microscope that has been able to detect single molecules. TopoMetrix: (408)982-9700, Fax: (408)982-9751

- Virtual Laboratories released a Phase Diagram Editor called P.D. Edit. The program allows users to draw phase diagrams and import the diagrams into their diffraction simulation and analysis program, Desktop Microscopist. The company also announced an internet e-mail address, and plans to set up a World Wide Web site in October, through which users can download demos, upgrades and the phase diagram editor, and ultimately exchange phase diagrams. Virtual Laboratories: (505)828-1640, Fax: (505)822-9759.

- Ronald Vane of XEI Scientific exhibited his SEM-CLEAN system for the first time at the MSA and MAS Conferences. He reports that there have been 76 SEM-CLEAN systems shipped to customers in the U.S. and Europe. XEI Scientific: (415)369-0133, Fax: (415)363-1659. 


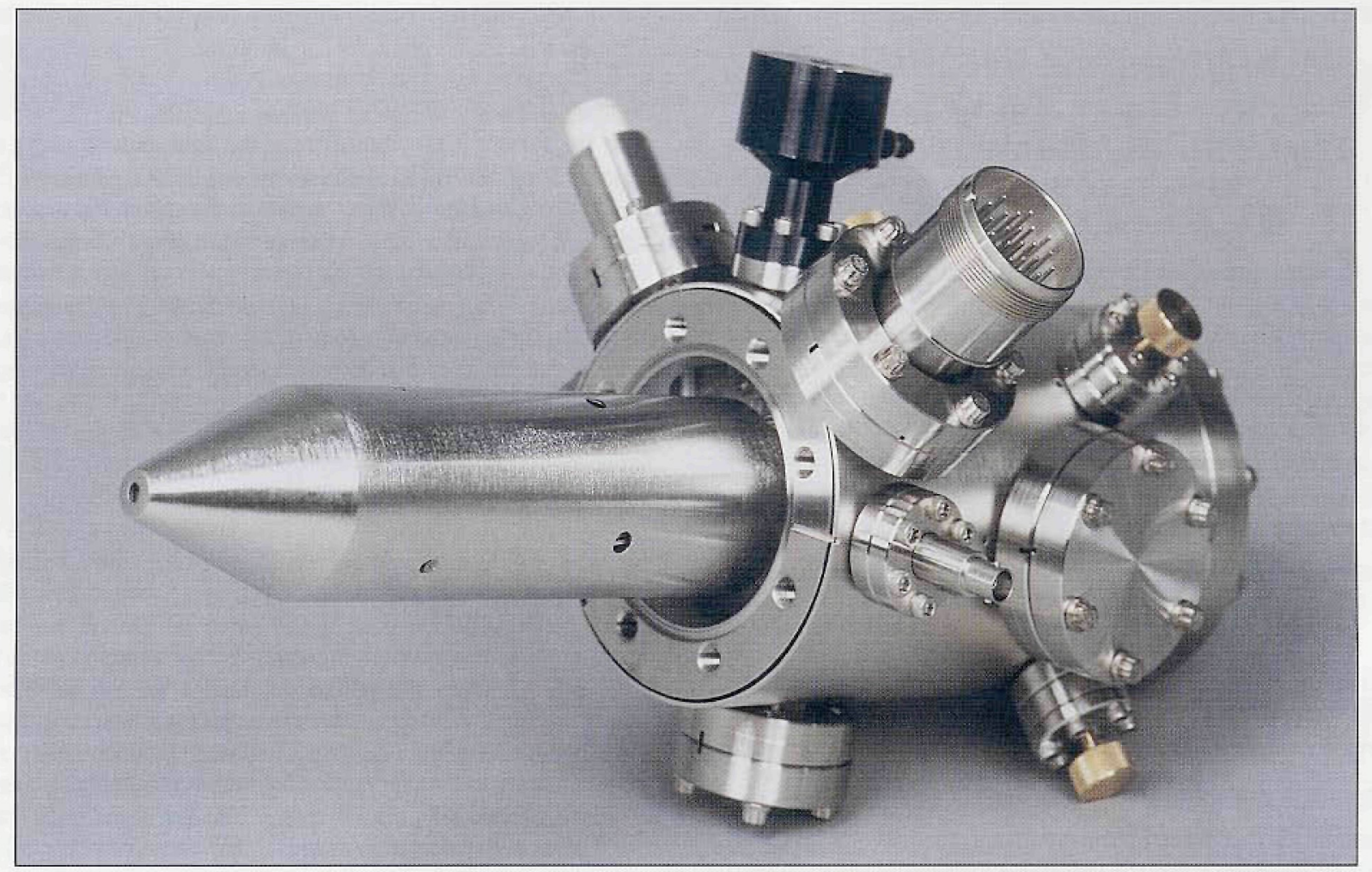

\section{What does fi stand for?}

Our compact, UHV, field emission columns are used by researchers world wide. Innovative electrostatic optics and dedicated electronics allow you to integrate a high current density electron or ion column into most vacuum systems. FEI also supplies researchers with other specialized products...

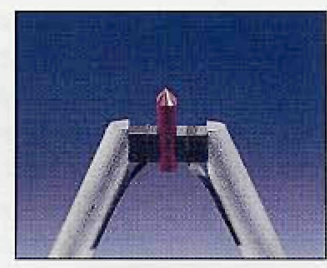

\section{$\mathrm{LaB}_{6}$ an $\mathrm{CeB}_{6}$ Cathodes}

FEl's Mini-Vogel Mount, the first universally compatible long-life, high-stability LaB6 cathode, provides excellent performance and the best cost-per-use value for installation into your EM systems.

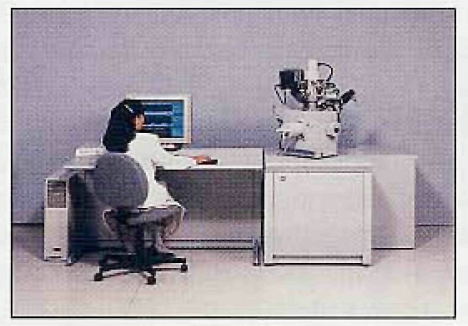

\section{FIB Workstations}

Focused ion beam micromilling workstations range from the 8"-wafer compatible model to the economical 2 " small sample model ideal for semiconductor, biological, TEM, and MEMS specimens.

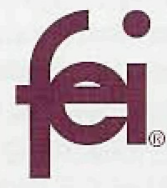

FEI Company 7451 NE Evergreen Parkway Hillsboro, OR $97124-5830$

(503) 640-7500 Fax (503) 640-7509

email:rsk@feico.com

Now, when you think of FEI, you'll know we are the Specialists in Field Electron and Ion Technology. 